\title{
Hyperhomogeneity of hydrogen-disordered ice and its origin: the residual entropy compatible with the disparity in hydrogen bond energy
}

Masakazu Matsumoto ( $\square$ matsu-m3@cc.okayama-u.ac.jp )

Okayama University https://orcid.org/0000-0002-6799-6813

Takuma Yagasaki

Okayama University

Hideki Tanaka

Okayama University

Article

Keywords: cryospheric science, thermodynamics, nonlinear dynamics

Posted Date: January 22nd, 2021

DOI: https://doi.org/10.21203/rs.3.rs-143208/v1

License: (c) (i) This work is licensed under a Creative Commons Attribution 4.0 International License.

Read Full License 


\title{
Hyperhomogeneity of hydrogen-disordered ice and its origin: the residual entropy compatible with the disparity in hydrogen bond energy
}

\author{
Masakazu Matsumoto ${ }^{1, *}$, Takuma Yagasaki ${ }^{2}$, and Hideki Tanaka ${ }^{1}$ \\ ${ }^{1}$ Research Institute for Interdisciplinary Science, Okayama University, Okayama 700-8530 Japan \\ ${ }^{2}$ Division of Chemical Engineering, Graduate School of Engineering Science, Osaka University, Osaka 560-8531 \\ Japan \\ *Author to whom correspondence should be addressed: vitroid@gmail.com
}

The residual entropy is one of the most crucial properties for the existence of a large number of ice polymorphs. The residual entropy has been estimated by Pauling assuming that there is no large difference between the hydrogen bond energies in ice. This simple model accurately predicts the entropy change of the phase transition between a hydrogen-disordered ice phase and its hydrogen-ordered counterpart. This fact is, however, incompatible with another fact that the difference in the pair interaction energies involved in hydrogen bonds in an ice phase can be larger than the thermal energy of a few $\mathrm{kJ} / \mathrm{mol}$. Here we propose a mechanism that reconciles them by considering the equality of the binding energy in each molecule rather than the pair interaction energy of the proximate pair. The topological feature of ice, called the ice rules, allows us to replace the interactions of a water molecule with the other individual molecules by that with the collections of the dipoles represented by directed cycles consisting of $\mathrm{O}-\mathrm{H}$ vectors. This resummation reveals that molecular environments in ice are extremely homogeneous thereby providing a solid basis for Pauling's model.

Unlike normal crystals, the crystal structure of ice has randomness regarding the orientation of hydrogen bonds. Most of the ice polymorphs with hydrogen-disorder transform into hydrogen-ordered counterparts when they are cooled. The difference in the entropy between the two phases, called residual entropy, ${ }^{1}$ is successfully calculated by Pauling's model, ${ }^{2}$ despite the fact that magnitude of the pair interaction depends significantly on the mutual orientation of the hydrogen-bonded dimer. ${ }^{3,4}$ Consequently, we arrive at a conjecture that the binding energy of a water molecule in hydrogen-disordered ice is almost the same for all molecules irrespective of the large difference in the pair interactions. 


\section{Results}

\section{Hyperhomogeneity of the binding energy}

Computer modelling allows us to estimate the intermolecular interaction between water molecules, which is capable of reproducing large differences in hydrogen bond energy in ice form. ${ }^{4-6}$ We show the distribution functions of the interaction energy of a water molecule with nearest neighbors in the leftmost panels of Fig. 1a for various ice polymorphs at $0 \mathrm{~K}$. The standard deviation (SD) of the interaction energy is 7 to $14 \mathrm{~kJ} \mathrm{~mol}^{-1}$ for ice $\mathrm{Ih}, \mathrm{III}, \mathrm{V}$, and $\mathrm{VI}$, and $\sim 20 \mathrm{~kJ} \mathrm{~mol}^{-1}$ for ice VII. These SD values seem too large to consider that all water molecules are energetically equivalent compared with the magnitude of the hydrogen-bonding energy, $\sim 25 \mathrm{~kJ} \mathrm{~mol}^{-1}$. The large energy discrepancies in ice manifest the experimental observation and theoretical calculation that the molecular positions are irregularly deviated from the lattice point due to the influence of the orientation of surrounding molecules, which is known as site-disorder of ice. ${ }^{6-9}$

The cumulative sum of the interaction energies of a water molecule with surrounding molecules is defined as

$$
I_{i}(r)=\sum_{j \mid r_{i j}<r} \phi\left(r_{i j}\right)
$$

where $\phi$ the pair interaction energy between water molecules $i$ and $j$ and the summation runs over all the molecules within a distance, $r$, from the central molecule, $i . I_{i}(\infty)$ is called the binding energy. The cumulative interactions, $I_{i}(r)$, of various ice types are plotted in the main panels of Figure 1a. In general, the standard deviation of the sum of random and statistically independent values must increase with increasing the number of the accumulated samples (see Figure S1 of SI). The width of the distribution decreases with increasing $r$ in Figure 1a. This indicates that there are some correlations between the interactions with nearest neighbors and the interactions with distant molecules as if the molecules in the middle to long range are oriented in such a way that they counteract the deviation by the interaction energy with the proximate environment. The SD of $I_{i}(r)$ at distance $r, \sigma(r)$, converges to a very small value at a long distance of $\sim 5 \mathrm{~nm}$ for all ice phases as shown in Fig 1b. The correlation is quite long-ranged for such a molecular system. The behavior of ice shown in Figure 1 can be called hyperhomogeneity. 


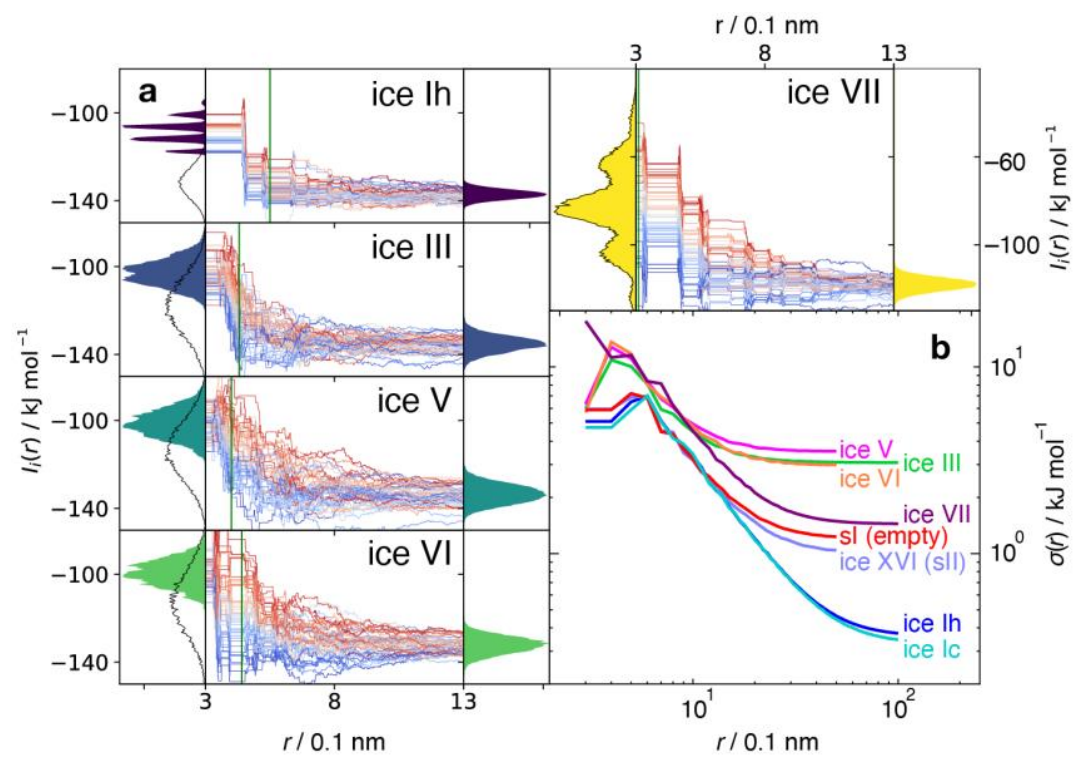

Figure 1 (a) Middle panels: Interaction energy of a water molecule with surrounding molecules summed over $r$, $I_{i}(r) . I(r)$ of fifty molecules are plotted for each ice type. The color of each curve is determined by the value of $I(r)$ at $0.4 \mathrm{~nm}$. Left and right panels: Distributions $I_{i}(r)$ at $\mathrm{r}=0.3$ and $1.3 \mathrm{~nm}$. The distance at which the width of the distribution is maximized, $r_{1}$, is indicated by a vertical green line and the distribution at the distance is shown with a thin black line in the left panel. (b) The standard deviation of the cumulative interaction of a water molecule with surrounding molecules, $\sigma(r)$, in various ice phases. Ice XVI and the empty sI are the clathrate hydrates without guests.

\section{Representation by a directed graph}

We explore the mechanism of the hyperhomogeneity in the hydrogen-disordered ice only in terms of Coulombic interactions between point charges located on atoms for simplicity. In ice, every water molecule accepts two hydrogen bonds and donates two hydrogen bonds. In graph theory, such a network can be represented by a simple regular directed graph with in- and out-degrees of two. A directed edge of the graph corresponds to a hydrogen bond and a vertex represents the position of the negative charge in a water molecule. ${ }^{5}$ A schematic diagram of the twodimensional model ice is shown in Figure 2a. There are numerous directed cycles in the graph of ice. A directed cycle is a cycle whose edges are traversed in the same direction. (In two dimensions, they are limited to clockwise or counterclockwise directions.) In hydrogen-disordered ice, the variety of the molecular arrangements in ice can be characterized by the spatial distribution of the directed cycles, just like the case of ice polymorphism where the structures of ice are characterized by the spatial arrangements of the undirected cycles (rings) in the hydrogen-bond network. ${ }^{10}$ We focus on the directed cycles to examine the effect of hydrogen-disordering. All 4-membered and 6membered directed cycles in the model ice are shown in Figures $2 \mathrm{~b}$ and 2c. One can find many other larger cycles in the graph. Here we deal with irreducible cycles only; we get rid of any cycles that pass through the same vertex 
more than once because they are reducible to smaller cycles.(Figure 2b) We also eliminate the cycle that spans the entire cell, that is essential for an ice to be polarized. ${ }^{11}$

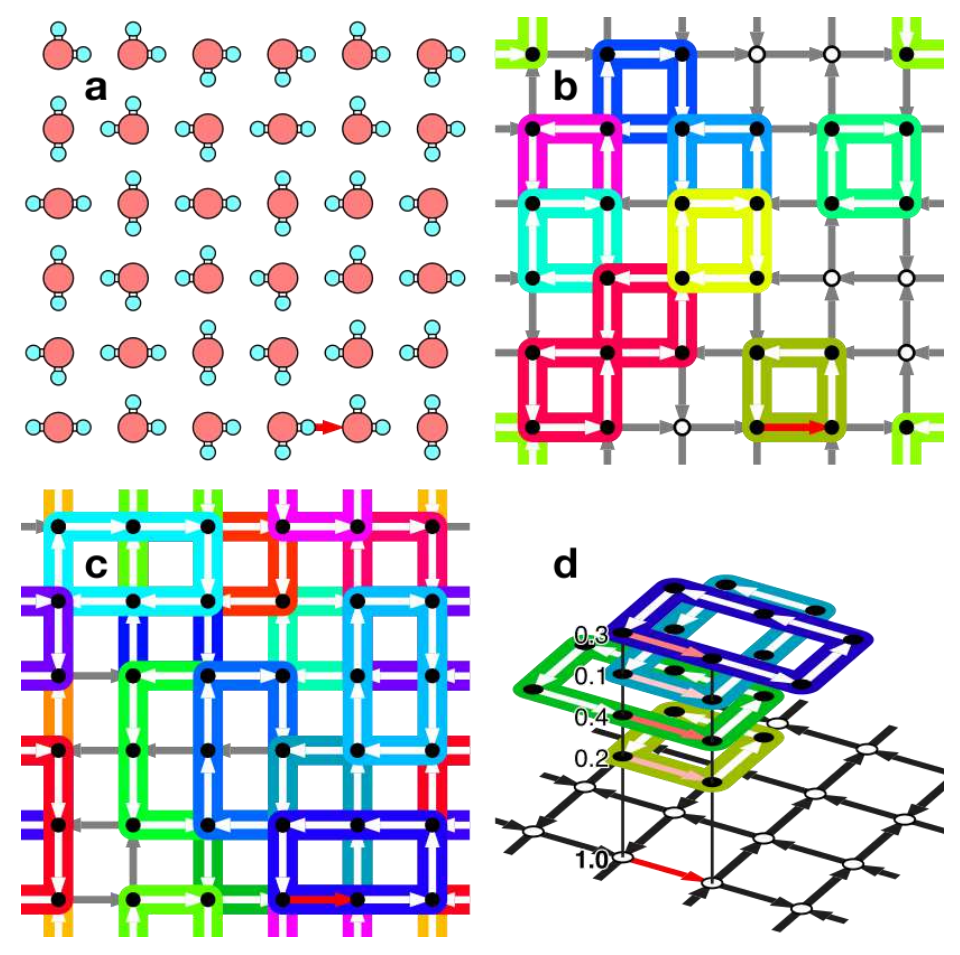

Figure 2. Schematic representation of the hydrogen-bonded network structure of ice. (a) The molecular configuration in ice (b) (c) The corresponding directed graph. All 4- and 6-membered directed cycles are painted in different colors. The red " 8 "-shaped 7-membered cycle in (b) is reducible to two 4-membered cycles, therefore we do not count it as a 7-membered cycle. (d) All small directed cycles that the red edge belongs to, and their respective weights.

\section{Interaction energy with cycles}

Each water molecule can also be viewed as being made up of two oxygen-to-hydrogen dipoles. In this paper, we will take this view to treat the two dipoles in a water molecule separately. The moments of all dipoles are identical. In general, the sum of a series of vectors that make up a ring is zero. Because the hydrogen bonds in each ice are all roughly equal in length and the $\mathrm{O}-\mathrm{H}$ dipoles are parallel to the O-O vectors, the net dipole moment of a directed cycle of hydrogen bonds is approximately zero. The interaction between a water molecule and a directed cycle in ice can be reduced to the dipole-quadrupole interaction, which is much short-ranged compared with the dipole-dipole interaction. 
We attempt to remove all dipole-dipole interactions by introducing the directed cycles. It can be achieved by an appropriate resummation of the interactions by the simplest and most tractable way. We enumerate all the cycles not larger than $n$-membered and make a set of them. The minimum size of the cycle depends on the type of ice, and the maximum size, $n$, is determined by the following procedure.

Let the set of all directed cycles up to $n$-membered ones in a given digraph be $C=\left\{c_{1}, c_{2}, \cdots\right\}$. A subset of $C$ consists of cycles passing through the edge $e_{i}$ is denoted by $C_{i}$. If one sets $n$ appropriately to find a sufficiently large number of cycles, the weights $w_{j}$ for the cycle $c_{j}$ are determined such that

$$
\sum_{j \mid c_{j} \in C_{i}} w_{j}=1
$$

exactly for all edges. (Figure 2d) To this end, all edges must belong to one or more cycles. In the case of Figure 2, all 4-membered and 6-membered cycles are sufficient to incorporate all the edges in the graph and to determine the weights. We increase $n$ one by one until appropriate $w$ is obtained. For ice Ih, $n=12$ is sufficient in most cases (it also depends on the molecular arrangements in the ice structure). A directed graph of ice is expressed by a linear combination of directed cycles contained in $C$. (The procedure to determine the weights is described in Methods.) That is, the interaction of a dipole with other dipoles is replaced by the weighted sum of the interactions of the dipole with cycles. It should be noted that a set of weights exactly satisfying eq. 3 can be obtained only for ice structures without polarization nor defects.

We calculate the cumulative interaction between water molecule $i$ and cycles located within a distance $r$ from the water molecule:

$$
I_{i}^{c}(r)=\sum_{j \mid r_{i}\left(c_{j}\right)<r} w_{j} \psi_{i j}
$$

where $r_{i}\left(c_{j}\right)$ is the distance between the vertex $i$ and directed cycle $j$, which is defined as the shortest one among the distances between the vertex $i$ and any vertex in cycle $j$. The distance is defined to be zero when the vertex $i$ is a member of cycle $j . \psi_{i j}$ is the sum of the Coulombic interactions between molecule $i$ and all the dipole vectors composing directed cycle $j$. As seen in Figure 3, the cumulative interaction at $r=0$ is close to that at long distances, indicating that the binding energy of a water molecule in ice is mostly determined by the interactions with the dipole vectors that share the same cycles with the central water molecule. As shown by the blue curves, the SD of the interaction energy decreases with increasing $r$ when the molecule-molecule interactions are accumulated. In contrast, as shown by the orange curves, the SD increases slightly with $r$ beyond $0.7 \mathrm{~nm}$ when the molecule-cycle interactions are accumulated. This suggests that there are no correlations even at $r=0.7 \mathrm{~nm}$ between moleculecycle interactions. 


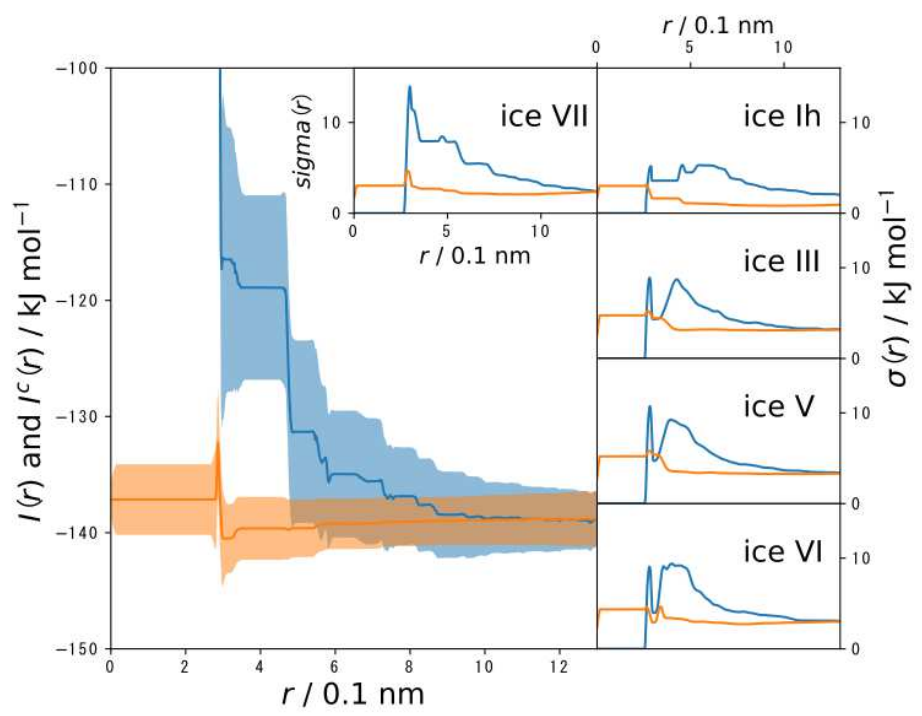

Figure 3. Cumulative molecule-cycle Coulombic interactions, $I^{\mathrm{c}}(r)$ (orange), and molecule-molecule Coulombic interactions, $I(r)$ (blue), for ice VII. The curve is the average over all molecules and the band indicates the standard deviation, $\pm \sigma$. The inset and side panels show the SDs, $\sigma(r)$, of $I(r)$ and $I^{c}(r)$ for several ice phases.

Cycles are classified into three categories for each central molecule: (a) cycles including the central molecule (Figure S2 of SI), (b) cycles incorporating the first adjacent molecule of the central molecule, and (c) the others. The distributions of the weighted sums of the interactions between the central molecule and the three types of cycles are plotted in Figure S3 of SI. The cycles containing the central molecule interact strongly with the central molecule. As shown in Figure S4 of SI, the strong interaction of this type of cycle is equivalent regardless of the number of edges in a cycle. This fact guarantees that the interaction with these cycles is equivalent irrelevant to the arrangement of the surrounding hydrogen bonds. Figure S3 demonstrates that the sum of the interaction energies of a water molecule with cycles (a) and cycles (b) is almost the same as the whole potential energy of the water molecule and the contribution from cycles (c) is negligible.

\section{Effects of Defects}

Water molecules at an interface or defect cannot be a member of a directed cycle. The interaction of such a molecule with another molecule is a dipole-dipole interaction, which is long-ranged compared with the interaction between a water molecule and a directed cycle. As a result, the distribution of the accumulated interaction energy becomes wide. Figure 4 shows the cumulative interaction of water molecules for the ice slab in vacuum. The SD of the cumulative interaction remains large for water molecules near the ice/vapor interface. The convergent tendency of the cumulative interactions recovers as we move from the surface to the interior of ice. 


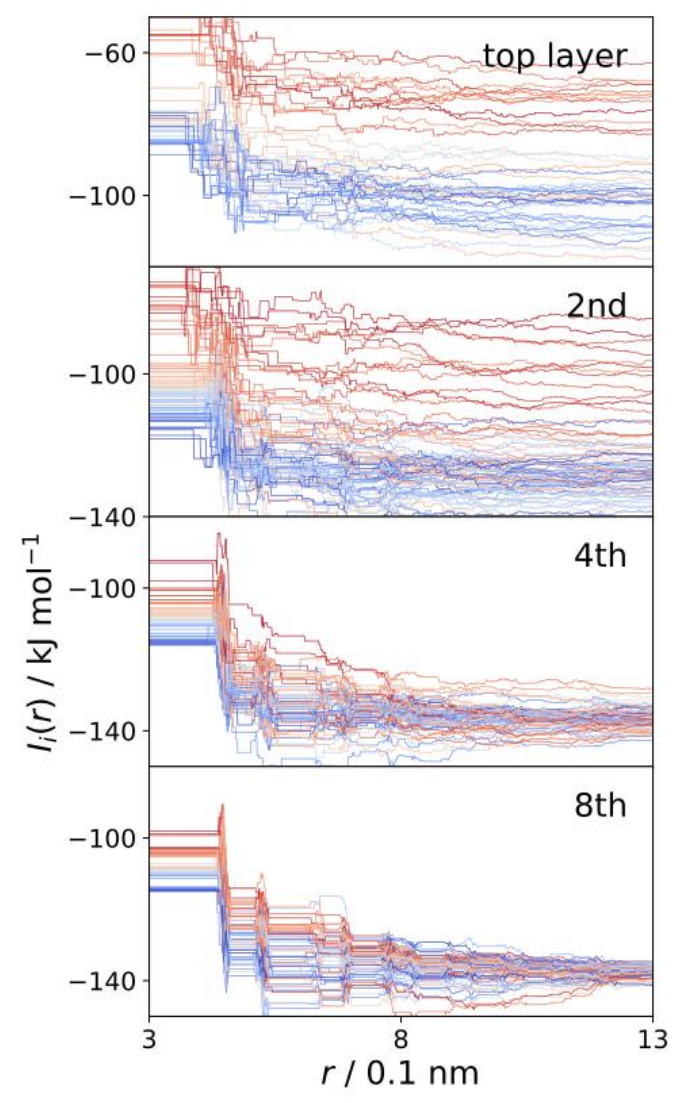

Figure 4. Cumulative interaction, $I_{i}(r)$, from the molecules at the surface of ice Ic. Each of the 32 molecules (16 on each of the top and bottom surfaces) from the top surface layer to the eighth layer (a layer is $0.16 \mathrm{~nm}$ thick) of the single crystal is selected as the central molecule, and the cumulative interaction, $I_{i}(r)$, with the remaining molecules is shown. The top layer is high in energy due to the lack of hydrogen bonds.

Figures $1 \mathrm{~b}$ show that the SD of ices at a long distance differ for different types of ice. The SD is larger for ice structures with a larger number of local environments for each molecule. For example, Ice III has two types of nonequivalent lattice points in the unit cell. In addition, there are six possible orientations of the water molecule at each position, some of which are equivalent reflecting the symmetry, and there are totally ten crystallographically distinct arrangements. The SD of each of ten types of water molecules is close to that of ice Ih (Figure S5 of SI). The SD is also larger for ice VI and VII with an interpenetrating double network. This is because there are no hydrogen bonds between the two networks to constrain the orientation of the molecules, and therefore strong attractive and repulsive forces are exerted depending on the orientation of the proximate molecules. This is consistent with the occurrence of site disorder in these ices. ${ }^{7}$ 


\section{Discussion}

We find a hyperhomogeneity in hydrogen-disordered ice that reconciles the local heterogeneity called the site disorder with Pauling's model to evaluate the residual entropy by the small disparity in the binding energy. The distribution function of the interaction energy between a water molecule and nearby water molecules is fairly wide. The corresponding distribution becomes substantially narrow when every water molecule is divided into two dipoles and the interactions of a molecule with other molecules are replaced by the interactions with directed cycles consisting of the dipoles. Such a resummation is allowed due to the ice rules: the hyperhomogeneity is an intrinsic property of ice.

There are efficient ways of accumulation to obtain the Madelung constant for an ionic crystal. If the Coulombic interactions are accumulated in an inappropriate order, the values will vary very widely depending on the order of summation, but the final value should be identical. ${ }^{12}$ Similarly, we find that there is an efficient way of accumulation to obtain a well converged potential energy of a water molecule in hydrogen-disordered ice. We demonstrate that the molecule-cycle interaction is strong when the cycle includes the water molecule. The contribution from the other cycles is quite small and only acts as a noise. On the other hand, the SD of the cumulative interactions becomes very large in the medium distance and indicates a slow convergence if the interactions are accumulated molecule by molecule in order of proximity.

It is expected that there are large energetic frustrations associated with the energy inequality due to the absence of directed cycles at interfaces and near impurities. Such frustrations may trigger a solid-solid phase transition such as hydrogen-ordering in ice Ih that occurs only when specific electrolytes are doped. ${ }^{13-16}$ The orientation of water molecules at the ice surface has been observed by advanced spectroscopic methods such as SFG (sum-frequency generation). ${ }^{17}$ When interpreting experimental measurements, one may assume implicitly that the water molecules at the surface are energetically equivalent. However, unlike inside the bulk ice, the distribution of the binding energies of water molecules is wide at the surface. Due to this heterogeneity, the Pauling model can break down for the ice surface and there may be significant biases in molecular orientation. ${ }^{18,19}$

The orientation of spins in magnetic substances called "spin ice" also obeys a rule that is topologically equivalent to the ice rules. ${ }^{20,21}$ There may be a similar effect on the spin-spin interactions in spin ices. The residual entropy is observed for crystalline carbon monoxide but there is no high order regularity that can correspond to the ice rules. (Figure S1 of SI) The hyperhomogeneity cannot exist for such a system.

\section{Methods}

\section{Modeling of Ice}

We prepare 30 hydrogen-disordered structures for each ice phase using the GenIce tool. ${ }^{11}$ All the structures obey the 
ice rule and have zero net dipole moment. The number of molecules in a cell is $\sim 1000$, which is large enough for the present analyses. The TIP4P/Ice water model is employed. ${ }^{5}$ The densities are set to $0.945,1.1655,1.2616,1.389$, and 1.688 for ices Ih, III, V, VI, and VII, respectively. Each structure is optimized at a fixed volume using the steepest descent energy minimization method. ${ }^{22}$

We generate an ice Ic slab structure in vacuum to examine the effect of the interface. The dimensions of the ice crystal consisting of 1024 water molecules are $2.55 \times 2.55 \times 5.1 \mathrm{~nm}$ and the dimensions of the simulation cell are $2.55 \times 2.55 \times 10 \mathrm{~nm}$. The structure is optimized using the steepest descent energy minimization method.

For long-ranged behaviors of SD appearing in Fig. 1b, 1,000 structures with $N=200,000$ to 400,000 for each crystalline form are generated and are subject to the steepest descent minimization of the total energy.

\section{Calculation of the weights}

The weight $w_{i}$ for each cycle is calculated as follows. First, all $2 N$ hydrogen bonds are labelled for an ice structure made of $N$ molecules. We search for all directed cycles of size $n$ or smaller and label them. The $i j$ element of a nonsquare matrix $\mathbf{A}$ is set to 1 if directed cycle $j$ contains hydrogen bond $i$, otherwise set to 0 . If the number of directed cycles is sufficiently greater than $2 N$, a columnar vector $\mathbf{w}$ satisfying $\mathbf{A} \cdot \mathbf{w}=\mathbf{1}$ can be determined by the pseudo inverse matrix method, where $\mathbf{1}$ is a $2 N$-row columnar vector in which all elements are 1 . A sample python code to calculate the weights of the directed cycles in a given structure of ice is provided in the GitHub repository: https://github.com/vitroid/CoverByCycles.

If the size $n$ of the cycle is infinitely large and the ice structure is free from defects and not polarized, it is easy to prove that there exists $\mathbf{w}$ that satisfies $\mathbf{A} \cdot \mathbf{w}=\mathbf{1}$. This is because it is always possible to draw a cyclic single stroke path that passes through all edges only once in the graph of such an ice structure, and the weight is unity. Another example is the case that the directed graph is tessellated into directed cycles where all the weights are unity (see Section S6 of SI).

\section{Data Availability}

In the interest of verifiable research, the data and code used to generate all Figures in both the main text and the supplementary information are available at https://github.com/vitroid/hyperhomogeneity. (Full disclosure will be made after acceptance of the manuscript.) 


\section{References}

1. Giauque, W. F. \& Ashley, M. F. Molecular Rotation in Ice at $10^{\circ}$ K. Free Energy of Formation and Entropy of Water. Phys. Rev. 43, 81-82 (1933).

2. Pauling, L. The Structure and Entropy of Ice and of Other Crystals with Some Randomness of Atomic Arrangement. Journal of the American Chemical Society vol. 57 2680-2684 (1935).

3. Kuhs, W. F. \& Lehmann, M. S. The Structure of Ice-Ih. Water Science Reviews 2 1-66 (1986) doi:10.1017/cbo9780511897504.001.

4. Kuo, J.-L., Klein, M. L. \& Kuhs, W. F. The effect of proton disorder on the structure of ice-Ih: a theoretical study. J. Chem. Phys. 123, 134505 (2005).

5. Abascal, J. L. F., Sanz, E., García Fernández, R. \& Vega, C. A potential model for the study of ices and amorphous water: TIP4P/Ice. The Journal of Chemical Physics vol. 122234511 (2005).

6. Knight, C. \& Singer, S. J. Site disorder in ice VII arising from hydrogen bond fluctuations. J. Phys. Chem. A 113, 12433-12438 (2009).

7. Kuhs, W. F., Finney, J. L., Vettier, C. \& Bliss, D. V. Structure and hydrogen ordering in ices VI, VII, and VIII by neutron powder diffraction. The Journal of Chemical Physics vol. 81 3612-3623 (1984).

8. Jorgensen, J. D. \& Worlton, T. G. Disordered structure of $\mathrm{D}_{2} \mathrm{O}$ ice VII from in situ neutron powder diffraction. J. Chem. Phys. 83, 329-333 (1985).

9. $\quad$ Nelmes, R. J. et al. Multisite Disordered Structure of Ice VII to 20 GPa. Phys. Rev. Lett. 81, 2719$2722(1998)$.

10. Salzmann, C. G., Radaelli, P. G., Slater, B. \& Finney, J. L. The polymorphism of ice: five unresolved questions. Phys. Chem. Chem. Phys. 13, 18468-18480 (2011).

11. Matsumoto, M., Yagasaki, T. \& Tanaka, H. GenIce: Hydrogen-Disordered Ice Generator. Journal of Computational Chemistry 39, 61-64 (2018).

12. Borwein, D., Borwein, J. M. \& Taylor, K. F. Convergence of lattice sums and Madelung's constant. 
J. Math. Phys. 26, 2999-3009 (1985).

13. Tajima, Y., Matsuo, T. \& Suga, H. Calorimetric study of phase transition in hexagonal ice doped with alkali hydroxides. J. Phys. Chem. Solids 45, 1135-1144 (1984).

14. Salzmann, C. G., Radaelli, P. G., Hallbrucker, A., Mayer, E. \& Finney, J. L. The preparation and structures of hydrogen ordered phases of ice. Science 311, 1758-1761 (2006).

15. Salzmann, C. G., Radaelli, P. G., Mayer, E. \& Finney, J. L. Ice XV: a new thermodynamically stable phase of ice. Phys. Rev. Lett. 103, 105701 (2009).

16. Köster, K. W., Klocke, T., Wieland, F. \& Böhmer, R. Interplay of defect doping and Bernal-Fowler rules: A simulation study of the dynamics on ice lattices. Physical Review B vol. 96 (2017).

17. Yamaguchi, S., Suzuki, Y., Nojima, Y. \& Otosu, T. Perspective on sum frequency generation spectroscopy of ice surfaces and interfaces. Chem. Phys. 522, 199-210 (2019).

18. Sugimoto, T., Aiga, N., Otsuki, Y., Watanabe, K. \& Matsumoto, Y. Emergent high-Tc ferroelectric ordering of strongly correlated and frustrated protons in a heteroepitaxial ice film. Nat. Phys. 12, 1063-1068 (2016).

19. Buch, V., Groenzin, H., Li, I., Shultz, M. J. \& Tosatti, E. Proton order in the ice crystal surface. Proc. Natl. Acad. Sci. U. S. A. 105, 5969-5974 (2008).

20. Isakov, S. V., Moessner, R. \& Sondhi, S. L. Why spin ice obeys the ice rules. Phys. Rev. Lett. 95, $217201(2005)$.

21. Harris, M. J., Bramwell, S. T., McMorrow, D. F., Zeiske, T. \& Godfrey, K. W. Geometrical Frustration in the Ferromagnetic PyrochloreHo2Ti2O7. Physical Review Letters vol. 79 2554-2557 (1997).

22. Stillinger, F. H. \& Weber, T. A. Hidden structure in liquids. Phys. Rev. A 25, 978-989 (1982). 


\section{Acknowledgments}

This work was supported by JSPS KAKENHI Grant Number $20 \mathrm{H} 05272$ and the Research Center for Computational Science in providing computational resources.

\section{Author Contributions}

M.M. noticed the problem and did the analysis; H.T. and T.Y. did the computations. All authors contributed to the discussion, construction of the logic, and writing of the manuscript. 


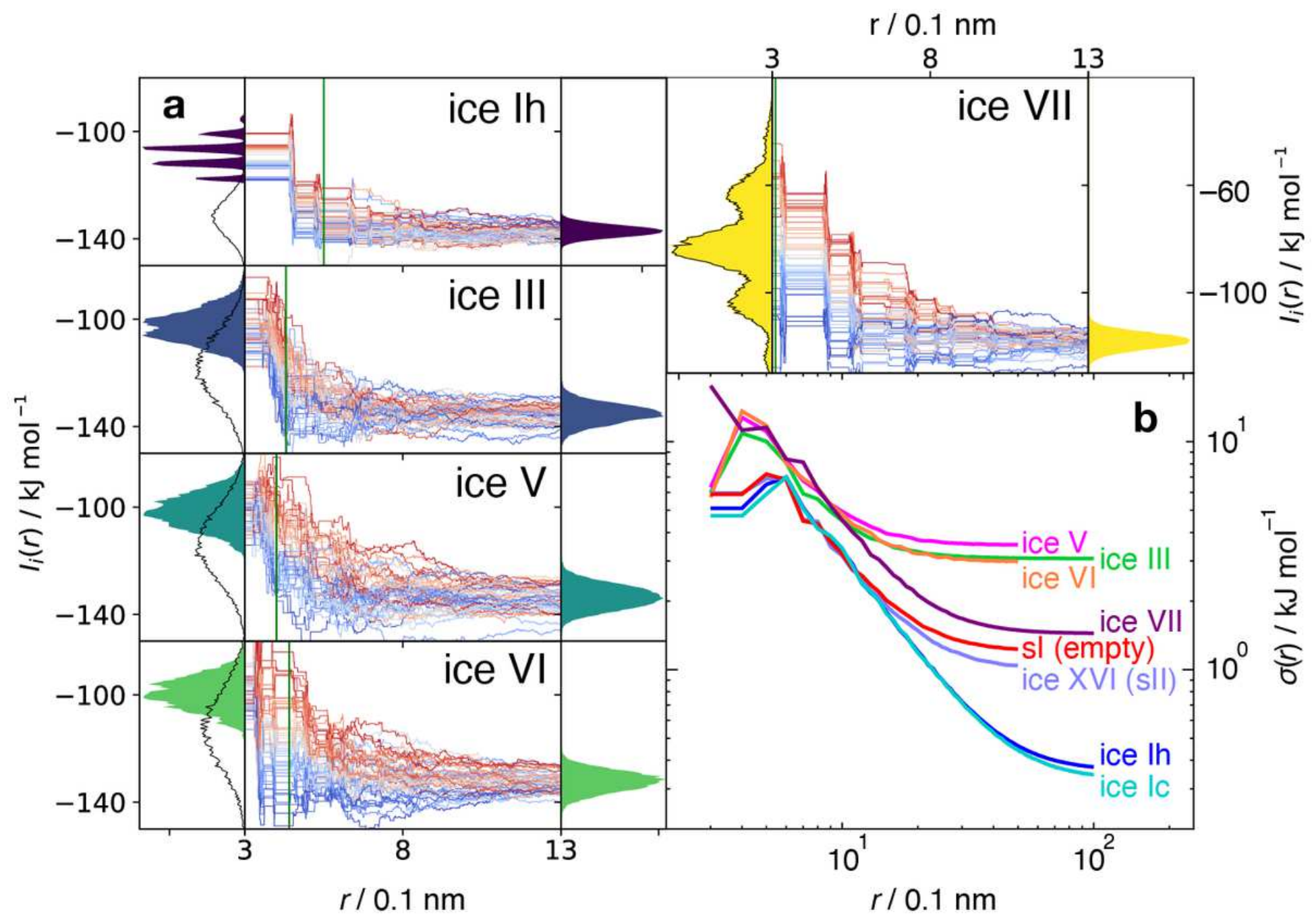

Figure 1

(a) Middle panels: Interaction energy of a water molecule with surrounding molecules summed over $r$, li(r). I(r) of fifty molecules are plotted for each ice type. The color of each curve is determined by the value of $\mathrm{I}(\mathrm{r})$ at $0.4 \mathrm{~nm}$. Left and right panels: Distributions li(r) at $r=0.3$ and $1.3 \mathrm{~nm}$. The distance at which the width of the distribution is maximized, $r 1$, is indicated by a vertical green line and the distribution at the distance is shown with a thin black line in the left panel. (b) The standard deviation of the cumulative interaction of a water molecule with surrounding molecules, $\sigma(r)$, in various ice phases. Ice XVI and the empty sl are the clathrate hydrates without guests. 


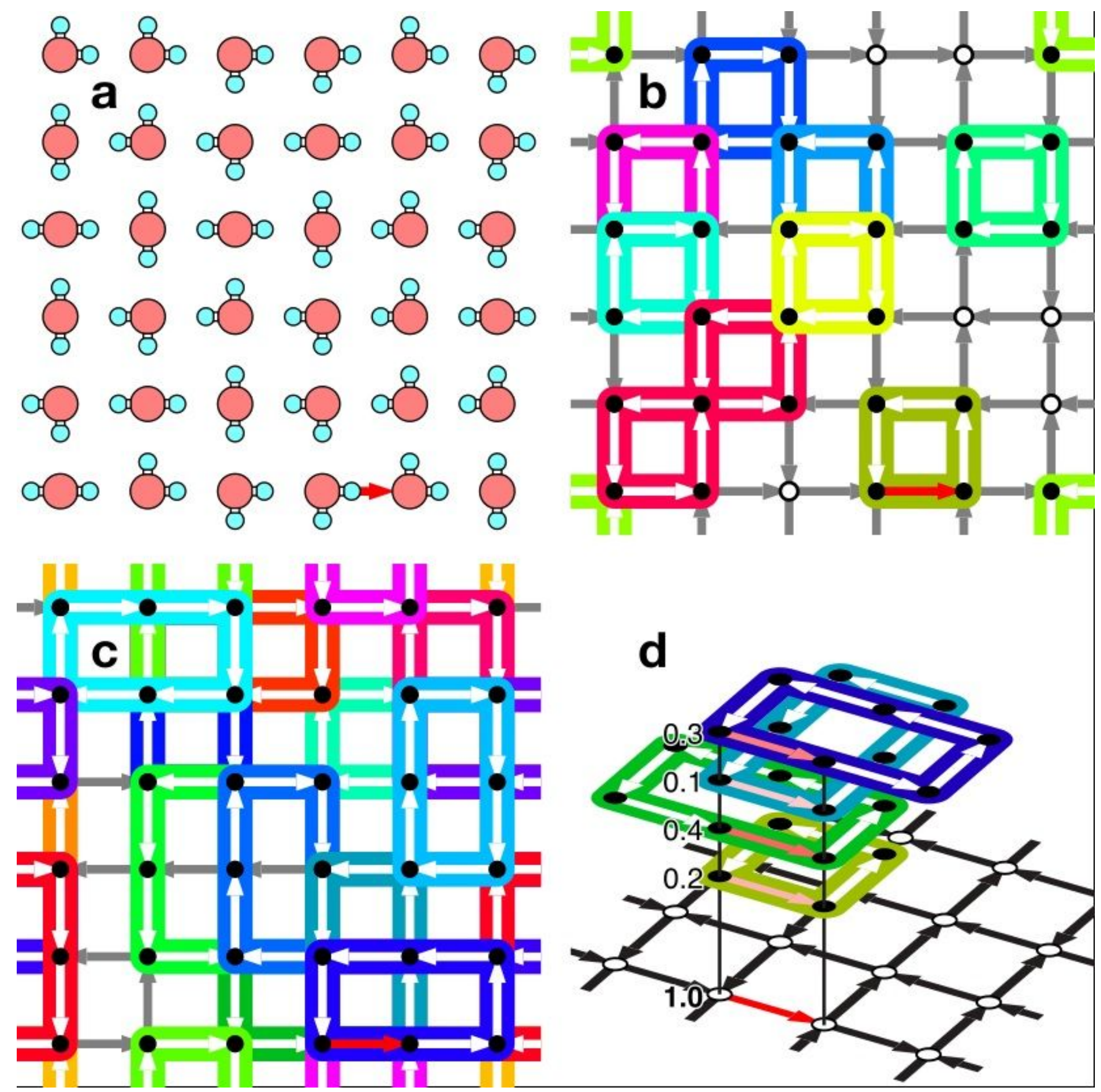

Figure 2

Schematic representation of the hydrogen-bonded network structure of ice. (a) The molecular configuration in ice (b) (c) The corresponding directed graph. All 4- and 6-membered directed cycles are painted in different colors. The red " 8 "-shaped 7-membered cycle in (b) is reducible to two 4-membered cycles, therefore we do not count it as a 7-membered cycle. (d) All small directed cycles that the red edge belongs to, and their respective weights. 


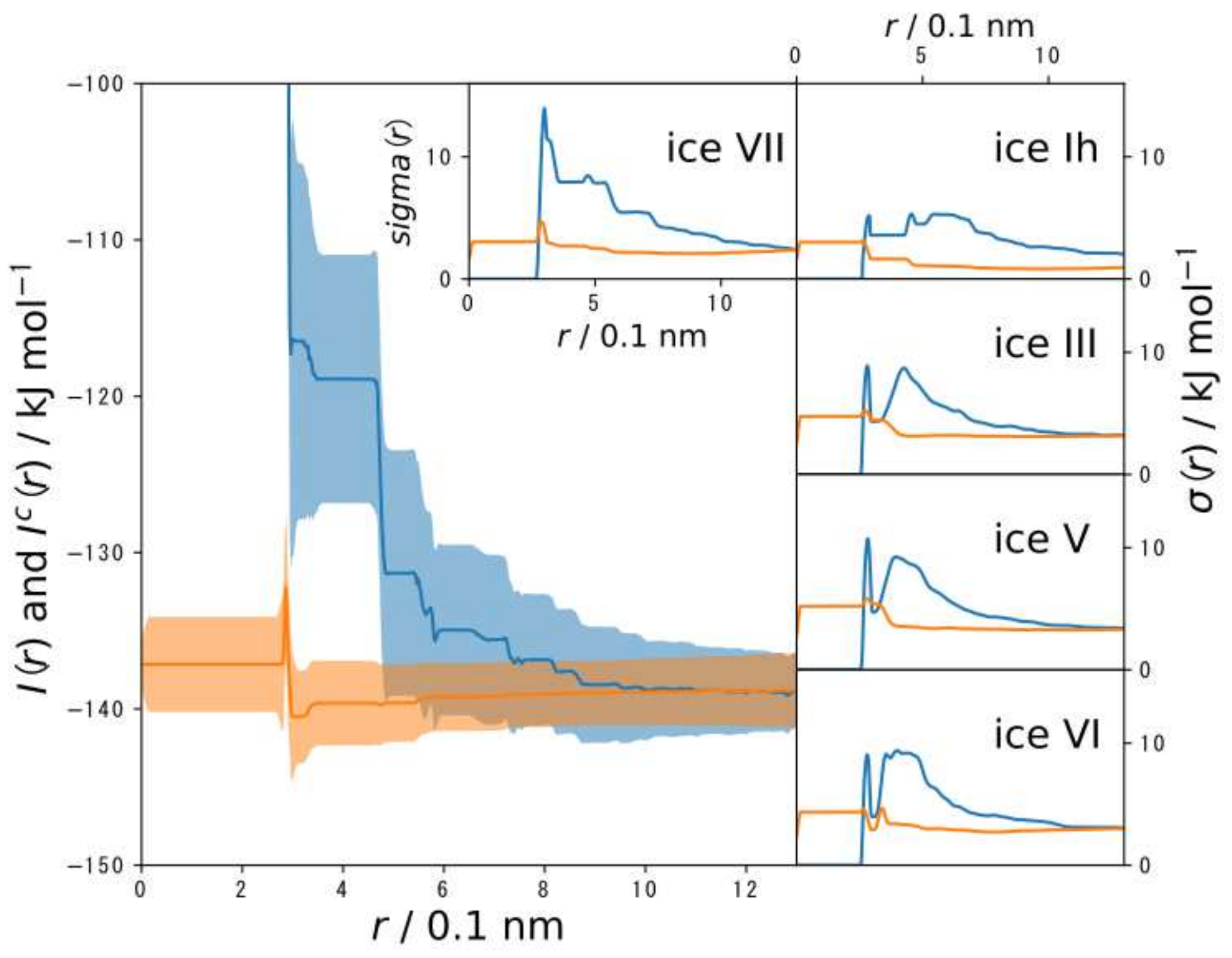

Figure 3

Cumulative molecule-cycle Coulombic interactions, Ic(r) (orange), and molecule-molecule Coulombic interactions, I(r) (blue), for ice VII. The curve is the average over all molecules and the band indicates the standard deviation, $\pm \sigma$. The inset and side panels show the SDs, $\sigma(r)$, of $I(r)$ and $I c(r)$ for several ice phases. 


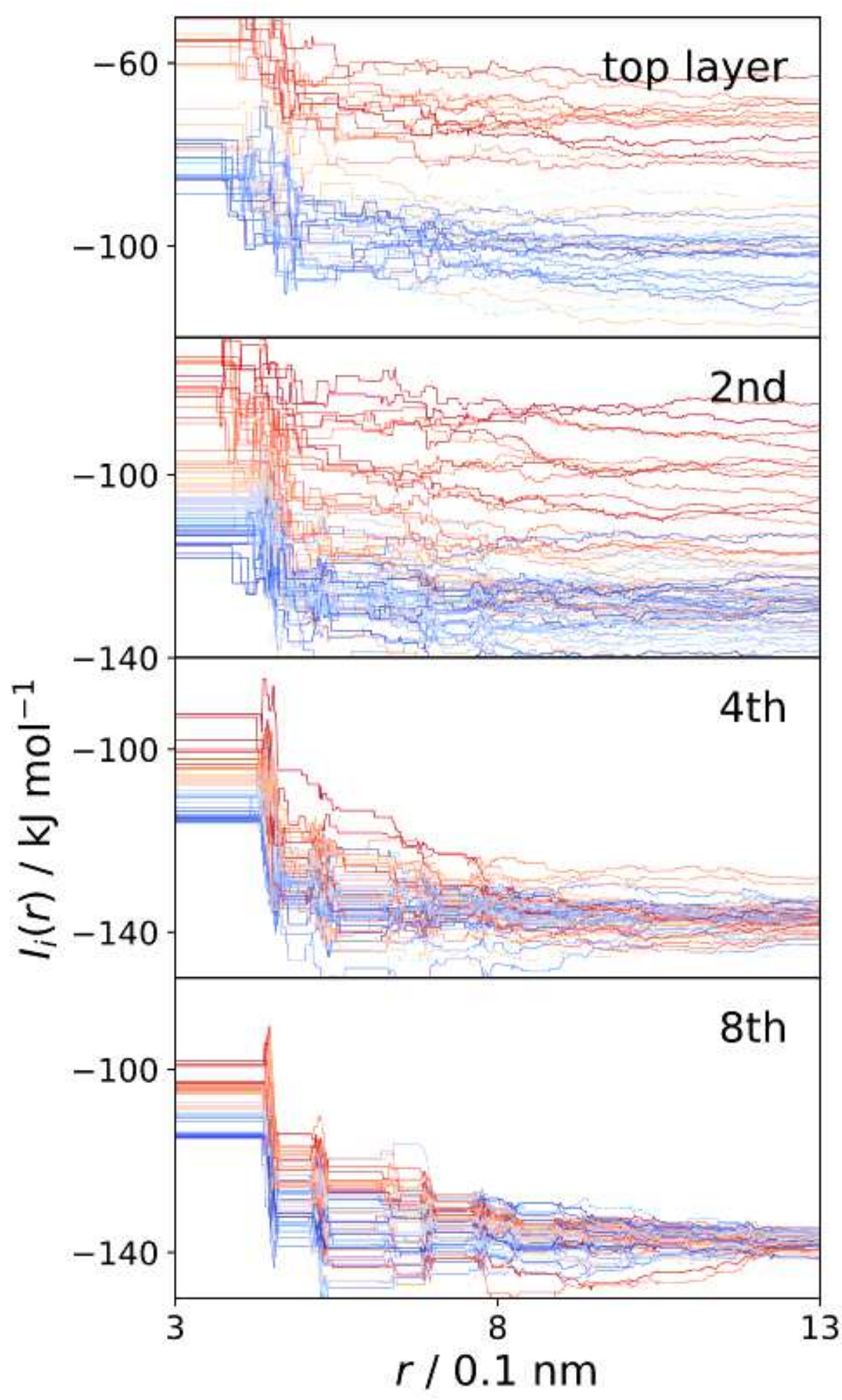

\section{Figure 4}

Cumulative interaction, li(r), from the molecules at the surface of ice lc. Each of the 32 molecules (16 on each of the top and bottom surfaces) from the top surface layer to the eighth layer (a layer is $0.16 \mathrm{~nm}$ thick) of the single crystal is selected as the central molecule, and the cumulative interaction, li(r), with the remaining molecules is shown. The top layer is high in energy due to the lack of hydrogen bonds. 


\section{Supplementary Files}

This is a list of supplementary files associated with this preprint. Click to download.

- supplement.docx 